\title{
Umbilical cord blood expansion with nicotinamide provides long-term multilineage engraftment
}

\author{
Mitchell E. Horwitz, ${ }^{1}$ Nelson J. Chao, ${ }^{1}$ David A. Rizzieri, ${ }^{1}$ Gwynn D. Long, ${ }^{1}$ \\ Keith M. Sullivan, ${ }^{1}$ Cristina Gasparetto, ${ }^{1}$ John P. Chute, ${ }^{1}$ Ashley Morris, ${ }^{1}$ Carolyn McDonald, ${ }^{1}$ \\ Barbara Waters-Pick, ${ }^{1}$ Patrick Stiff, ${ }^{2}$ Steven Wease, ${ }^{3}$ Amnon Peled,, ${ }^{4}$ David Snyder, ${ }^{5}$ \\ Einat Galamidi Cohen, ${ }^{5}$ Hadas Shoham, ${ }^{5}$ Efrat Landau, ${ }^{5}$ Etty Friend, ${ }^{5}$ Iddo Peleg, ${ }^{5}$ \\ Dorit Aschengrau, ${ }^{5}$ Dima Yackoubov, ${ }^{5}$ Joanne Kurtzberg, ${ }^{6}$ and Tony Peled ${ }^{5}$ \\ ${ }^{1}$ Adult Blood and Marrow Transplant Program, Duke University Medical Center, Durham, North Carolina, USA. \\ ${ }^{2}$ Loyola University Medical Center, Maywood, Illinois, USA. ${ }^{3}$ The EMMES Corporation, Rockville, Maryland, USA. \\ ${ }^{4}$ Goldyne Savad Institute of Gene Therapy, Hadassah - Hebrew University Medical Center, Jerusalem, Israel. ${ }^{5}$ Gamida Cell Ltd., \\ Jerusalem, Israel. ${ }^{6}$ Pediatric Blood and Marrow Transplant Program, Duke University Medical Center, Durham, North Carolina, USA.
}

\begin{abstract}
Background. Delayed hematopoietic recovery is a major drawback of umbilical cord blood (UCB) transplantation. Transplantation of ex vivo-expanded UCB shortens time to hematopoietic recovery, but long-term, robust engraftment by the expanded unit has yet to be demonstrated. We tested the hypothesis that a UCB-derived cell product consisting of stem cells expanded for 21 days in the presence of nicotinamide and a noncultured $T$ cell fraction (NiCord) can accelerate hematopoietic recovery and provide long-term engraftment.
\end{abstract}

Methods. In a phase I trial, 11 adults with hematologic malignancies received myeloablative bone marrow conditioning followed by transplantation with NiCord and a second unmanipulated UCB unit. Safety, hematopoietic recovery, and donor engraftment were assessed and compared with historical controls.

Results. No adverse events were attributable to the infusion of NiCord. Complete or partial neutrophil and $T$ cell engraftment derived from NiCord was observed in 8 patients, and NiCord engraftment remained stable in all patients, with a median follow-up of 21 months. Two patients achieved long-term engraftment with the unmanipulated unit. Patients transplanted with NiCord achieved earlier median neutrophil recovery (13 vs. 25 days, $P<0.001)$ compared with that seen in historical controls. The 1 -year overall and progression-free survival rates were $82 \%$ and $73 \%$, respectively.

Conclusion. UCB-derived hematopoietic stem and progenitor cells expanded in the presence of nicotinamide and transplanted with a $\mathrm{T}$ cell-containing fraction contain both short-term and long-term repopulating cells. The results justify further study of NiCord transplantation as a single UCB graft. If long-term safety is confirmed, NiCord has the potential to broaden accessibility and reduce the toxicity of UCB transplantation.

Trial Registration. Clinicaltrials.gov NCT01221857.

Funding. Gamida Cell Ltd.

\section{Introduction}

Delayed hematopoietic and immunologic recovery, graft failure, and graft versus host disease (GVHD) all contribute to transplantrelated mortality in adult recipients of umbilical cord blood (UCB)

Conflict of interest: Mitchell E. Horwitz has received research support from Gamida Cell, Sanofi, and Pfizer. Joanne Kurtzberg is a medical advisor for the National Marrow Donor Program Center for Cord Blood and StemCyte Cord Blood Bank, medical director of the Carolinas Cord Blood Bank and CORD:USE Cord Blood Bank, a member of the FACT board of directors, and a member of the Advisory Council of Blood Stem Cell Transplantation (Department of Health and Human Services). Tony Peled, David Snyder, Einat Galamidi Cohen, Hadas Shoham, Efrat Landau, Etty Friend, Iddo Peleg, Dorit Aschengrau, and Dima Yackoubov are employees of Gamida Cell.

Note regarding evaluation of this manuscript: Manuscripts authored by scientists associated with Duke University, The University of North Carolina at Chapel Hill, Duke-NUS, and the Sanford-Burnham Medical Research Institute are handled not by members of the editorial board but rather by the science editors, who consult with selected external editors and reviewers.

Citation for this article: J Clin Invest. 2014;124(7):3121-3128. doi:10.1172/JCI74556 transplantation (1-5). Cell dose and HLA matching are critical determinants of a successful outcome (6-9). For patients without a single UCB unit with adequate cell dose, dual UCB transplantation is an acceptable alternative (10). Yet, the problem of delayed hematopoietic recovery persists, leading to longer hospitalization and increased resource utilization. Ex vivo expansion of hematopoietic stem and progenitor cells (HSPCs) is a modality that could address these limitations of cord blood transplantation. If short-term and long-term HSPCs could be expanded ex vivo from UCB, then prompt and durable hematopoietic recovery after transplantation of a single UCB unit could be achieved in the majority of patients. Recently reported cord blood expansion studies have demonstrated the contribution of expanded cells to short-term engraftment, while long-term engraftment came from the coinfused second unmanipulated unit $(11,12)$.

NiCord is an ex vivo-expanded cell product derived from UCB that uses a small molecule, nicotinamide, as the active agent that 
Table 1

Patient and graft characteristics

\begin{tabular}{|c|c|c|}
\hline Characteristics & $\begin{array}{c}\text { Subjects } \\
n=11\end{array}$ & $\begin{array}{c}\text { Historical controls } \\
\qquad n=17\end{array}$ \\
\hline \multicolumn{3}{|l|}{ Age (years) } \\
\hline Median 45 & 31 & \\
\hline Range 21-61 & $23-55$ & \\
\hline \multicolumn{3}{|l|}{ Weight (kg) } \\
\hline Median 83 & 77 & \\
\hline Range 59-100 & $47-112$ & \\
\hline \multicolumn{3}{|l|}{ Diagnosis (\%) } \\
\hline AML or MDS & $8(73)$ & $10(58)$ \\
\hline ALL $\quad 1(9)$ & $4(24)$ & \\
\hline CML $\quad 0$ & $1(6)$ & \\
\hline Lymphoma & $2(18)$ & $2(12)$ \\
\hline \multicolumn{3}{|l|}{$\begin{array}{l}\text { Disease status at time } \\
\text { of transplantation (\%) }\end{array}$} \\
\hline \multicolumn{3}{|l|}{ Complete remission } \\
\hline First remission & $4(36)$ & $2(12)$ \\
\hline Second or subsequent remission & $3(28)$ & $12(71)$ \\
\hline Active disease & $4(36)$ & $3(17)$ \\
\hline \multicolumn{3}{|l|}{ Preparative regimen } \\
\hline TBI 1,350 cGy/fludarabine & 9 & 17 \\
\hline $\begin{array}{l}\text { TBI 1,350 cGy/fludarabine/ } \\
\text { cyclophosphamide }\end{array}$ & 2 & 0 \\
\hline \multicolumn{3}{|c|}{$\begin{array}{l}\text { Donor-recipient HLA compatibility } \\
\text { NiCord/unmanipulated + unmanipulated }\end{array}$} \\
\hline $6 / 6+6 / 6$ & 0 & 1 \\
\hline $5 / 6+6 / 6$ & 1 & 2 \\
\hline $5 / 6+5 / 6$ & 1 & 1 \\
\hline $4 / 6+5 / 6$ & 2 & 6 \\
\hline $5 / 6+4 / 6$ & 1 & 6 \\
\hline $4 / 6+4 / 6$ & 6 & 7 \\
\hline
\end{tabular}

ALL, acute lymphoblastic leukemia; AML, acute myeloid leukemia; CML, chronic myeloid leukemia; MDS, myelodysplastic syndrome.

inhibits differentiation and enhances the functionality of HSPCs expanded in ex vivo cultures. When nicotinamide is added to stimulatory hematopoietic cytokines, UCB-derived hematopoietic progenitor cell cultures demonstrate an increased frequency of phenotypically primitive $\mathrm{CD} 34^{+} \mathrm{CD} 38^{-}$cells and a decreased frequency of lineage-committed progenitor cells. The cells expanded in culture with nicotinamide demonstrate increased migration toward stromal cell-derived factor 1 and increased homing to the bone marrow, resulting in enhanced engraftment efficiency (13).

Double UCB transplantation provides a safe clinical experimental model to demonstrate the existence of short- and long-term repopulating hematopoietic stem cells as well as the clinical benefit of an ex vivo-expanded graft. We report here results of a phase I trial testing the hypothesis that NiCord can safely provide HSPCs that are capable of producing rapid and durable hematopoietic engraftment in adult recipients of myeloablative UCB transplantation.

\section{Results}

Patients. Twelve patients (Duke University Medical Center, 11 patients; Loyola University Medical Center, 1 patient) were enrolled in the study (Figure 1). One patient was successfully transplanted with a single unmanipulated cord blood unit, because the NiCord unit failed to meet release criteria (initial gram-positive stain, later deemed to be a false-positive). This patient was excluded from outcome analysis. Table 1 shows the characteristics of the 11 evaluable study patients and the 17 Duke historical control patients (14). All patients had hematologic malignancies in either complete or partial remission and received $1,350 \mathrm{cGy}$ total body irradiation (TBI) as part of the conditioning regimen.

Graft characteristics. Table 2 shows the cell doses of the unmanipulated and NiCord-designated UCB units before cryopreservation, as reported by the cord blood bank, and the cell doses and graft characteristics at the time of infusion. The median total nucleated cell dose of the unmanipulated unit and the NiCord unit was $2.6 \times 10^{7}$ (range, $1.9 \times 10^{7}$ to $4.3 \times 10^{7}$ ) per kilogram of the recipient's body weight and $2.5 \times 10^{7}$ (range, $1.7 \times 10^{7}$ to $3.8 \times 10^{7}$ ) per kilogram of the recipient's body weight, respectively $(P=0.28)$. The median $\mathrm{CD} 34^{+}$cell dose at the time of cryopreservation of the unmanipulated unit was $0.12 \times 10^{6}$ (range, $0.03 \times 10^{6}$ to $0.23 \times 10^{6}$ ) per kilogram of the recipient's body weight and $0.17 \times 10^{6}$ (range, $0.05 \times 10^{6}$ to $0.35 \times 10^{6}$ ) per kilogram of the recipient's body weight $(P=0.40)$ for the NiCord unit. The $\mathrm{CD} 133^{+}$fraction of the NiCord unit was cultured for 21 days, resulting in a median 486-fold expansion (range, 171-643) of the nucleated cells. When combined with the noncultured CD133- fraction, the median final infused total nucleated cell dose was $3.1 \times 10^{7}$ per kilogram. NiCord expansion resulted in a median 72 -fold (range, 16-186) expansion of CD34+ cells, providing a median infused $\mathrm{CD}_{3} 4^{+}$cell dose of $3.5 \times 10^{6}$ (range, $0.9 \times 10^{6}$ to $\left.18.3 \times 10^{6}\right)$ per kilogram. The median infused CD $34^{+}$cell dose from the unmanipulated unit was $0.07 \times 10^{6}$ (range, $0.03 \times 10^{6}$ to $\left.0.48 \times 10^{6}\right)$ per kilogram. The $\mathrm{CD}^{+}$cell dose from the NiCord unit was derived entirely from the noncultured CD133- fraction. As a consequence of multiple manipulations, the median $\mathrm{CD}^{+}$cell dose from the NiCord unit was $1.3 \times 10^{6}$ (range, $0.49 \times 10^{6}$ to $5.81 \times 10^{6}$ ) per kilogram, which was significantly lower than the median cell dose from the manipulated unit of $3.4 \times 10^{6}$ (range, $1.9 \times 10^{6}$ to $4.7 \times 10^{6}$ ) per kilogram $(P=0.009)$. Of note, the infused $\mathrm{CD}^{+}$cell dose was not directly measured; instead, it was estimated based on the experience accumulated during product development, which demonstrated a median $70 \%$ recovery after thawing.

Chimerism analysis. Figure 2 shows the pattern of engraftment within the $\mathrm{CD}_{15} 5^{+}$myeloid cell and $\mathrm{CD}^{+} \mathrm{T}$ cell fractions from peripheral blood for the 10 patients who were engrafted with donor cells. Patient 10 demonstrated $100 \%$ NiCord donor chimerism in samples collected from unfractionated whole blood through the final day of assessment (day 42). Lineage-specific chimerism was not available; however, it is assumed that the observed NiCord-derived cells were predominantly myeloid cells, given the early time points at which they were collected. Eight patients (patients 1, 2, 3, 6, 7, 9, 10, and 11) demonstrated a persistent presence of NiCord-derived myeloid cells ranging from $41 \%$ to $100 \%$. Stable engraftment of $\mathrm{T}$ cells derived from the NiCord unit was also observed in 6 patients (patients 2, 3, 6, 7, 9, and 11). Mixed hematopoietic chimerism (donor-donor or donor-host) was more common in the $\mathrm{T}$ cell fraction. In all surviving patients, NiCord engraftment has remained stable for up to 36 months, suggesting engraftment of NiCord-derived HSPCs. Two patients (patients 4 and 5) achieved long-term engraftment that was exclusively from 


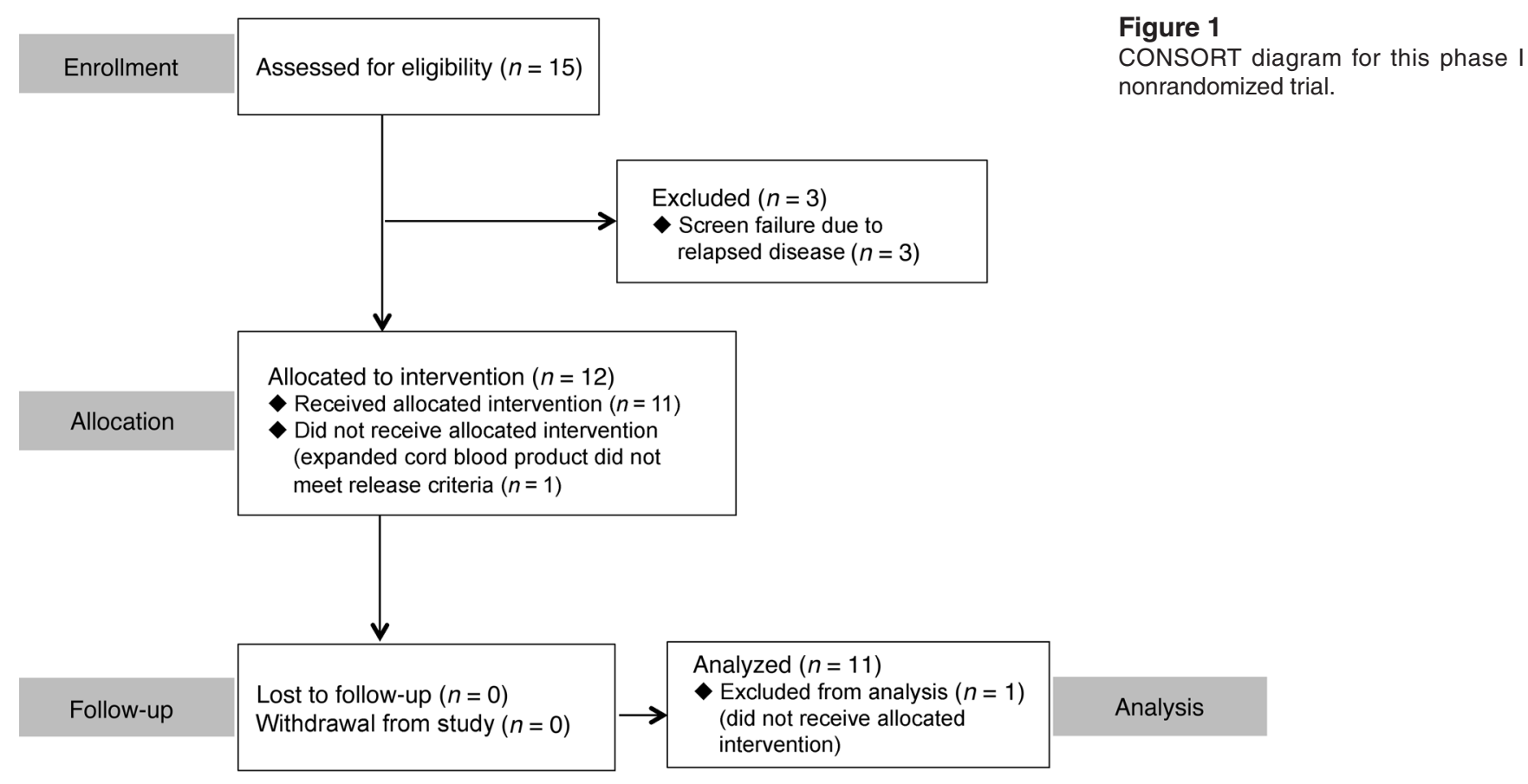

the unmanipulated unit. One patient (patient 8) failed to engraft and was successfully rescued with a second transplant from a haploidentical donor. The infusion order of NiCord and the unmanipulated unit did not correlate with the pattern of engraftment.

Hematopoietic recovery. The kinetics of white blood cell count recovery and the median time to neutrophil recovery are demonstrated in Figure 3A. For all patients transplanted with NiCord, neutrophil recovery was achieved in 13 days (range, 7-26 days) versus 25 days (range, 13-38 days) in the Duke historical cohort $(P<0.001)$. The median time to neutrophil recovery for the 8 patients engrafted with NiCord was 11 days (range, 7-18 days). At later time points out to 2 years, we found no significant difference in the white blood cell count of the study patients or of the Duke controls (Figure 3B).

The median time to platelet engraftment was 33 days (range, 26-49 days) for all patients transplanted with NiCord and 37 days (range, 20-66 days) for the Duke historical control cohort $(P=0.085)$ (Figure 3C). Platelet recovery occurred on median day 30 (range, 26-41) for the 7 patients who demonstrated engraftment with NiCord. Patient 10 died before achieving platelet recovery, and patient 8 experienced primary engraftment failure. At later time points out to 2 years, we observed no significant difference in the platelet counts of study patients or of Duke controls (Figure 3D). We have observed no secondary graft failure to date in any of the patients who achieved donor engraftment from either the unmanipulated or the NiCord-expanded cord blood unit.

For patients who were engrafted with the NiCord-expanded cord blood unit, the number of total nucleated cells contained within the product correlated with the speed of both neutrophil (Spearman's correlation coefficient, $-0.86 ; P=0.006$ ) and platelet engraftment (Spearman's correlation coefficient, -0.77 ; $P=0.04$ ). The number of $\mathrm{CD} 34^{+}$cells in the NiCord product also correlated with the speed of neutrophil (Spearman's correlation coefficient, $r=-0.82 ; P=0.01$ ) and platelet (Spearman's correlation coefficient, $r=-0.77 ; P=0.04$ ) engraftment.
Transplantation course and outcome. Transplantation course and outcome are summarized in Table 3. One grade III adverse event (hypertension) and no grades IV/V adverse events were attributable to infusion of the NiCord UCB graft. The median duration of initial hospitalization for all study patients was 26 days (range, 17-57 days).

We observed acute grade II GVHD in 5 patients and no cases of acute grades III/IV GVHD. Two patients developed chronic GVHD, 2 patients (patients 3 and 9) died of relapsed disease, and 1 patient (patient 10) died of pneumonia. With a median follow-up of 21 months, the 1-year overall and progression-free survival rates were $82 \%$ and $73 \%$, respectively (Figure 4 ).

\section{Discussion}

Based on preclinical work suggesting that HSPCs with increased capacity for bone marrow migration, homing, and engraftment can be expanded from UCB using NiCord technology (13), we performed a phase I clinical trial to confirm this observation. Using the myeloablative double cord blood transplantation approach $(15,16)$, we transplanted 1 unmanipulated unit and a second unit in which a CD $133^{+}$fraction was cultured ex vivo for 3 weeks and infused with a small, uncultured $T$ cell fraction. The cultured unit provided long-term engraftment in 8 of 10 evaluable patients. The novel finding of this trial is that an expanded UCB graft is capable of outcompeting an unmanipulated cord blood graft and of providing both rapid engraftment and robust, multilineage hematopoiesis for more than 2 years.

Following dual UCB transplantation, hematopoiesis is ultimately provided by a single UCB unit in nearly all cases. Factors that predict the dominant unit have yet to be fully characterized, but there is evidence that an immunologic "graft versus graft" reaction is initiated following transplantation that culminates in the elimination of the immunologically nondominant unit $(17,18)$. Nicotinamide, the active molecule of NiCord expansion technology, slows down differentiation of proliferating early 


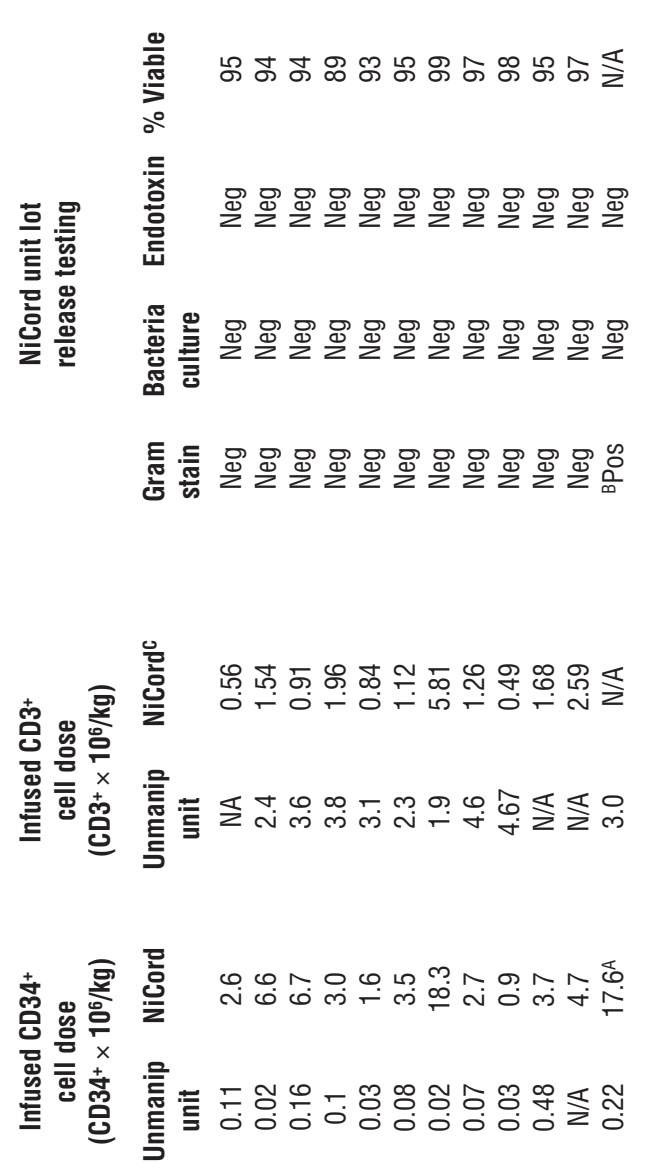

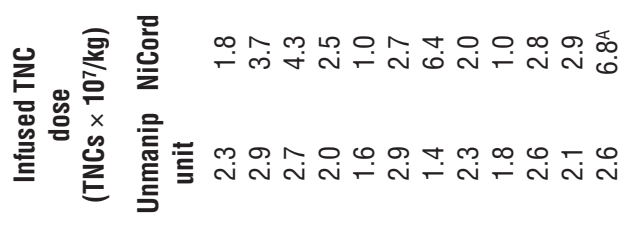
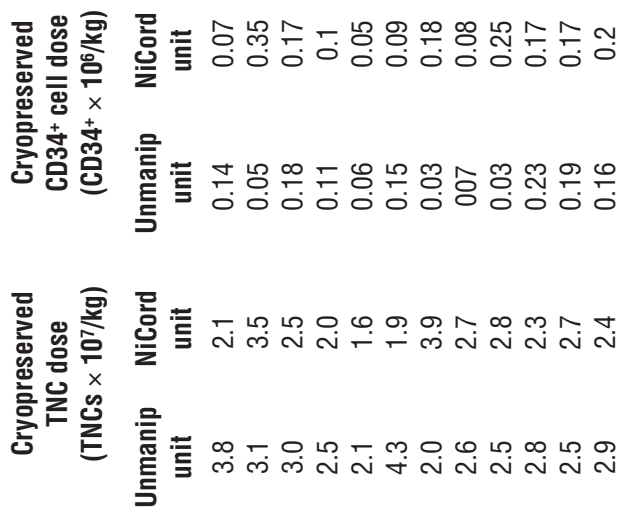

progenitor cells, thus enhancing expansion of primitive progenitor cells $\left(\mathrm{CD} 34^{+} \mathrm{CD} 38^{-}\right.$and $\left.\mathrm{CD} 34^{+} \mathrm{CD} 38^{-} \mathrm{Lin}^{-}\right)(13)$. The expanded fraction provides robust myeloid and $\mathrm{T}$ cell engraftment in immune-deficient NOD/SCID/IL-2 receptor $\gamma$ chain knockout (NSG) mice for more than 6 months (data not shown). However, NiCord culture conditions do not support the expansion of mature lymphoid cells. To compensate for this immunologic disadvantage, we coinfused the CD133- noncultured fraction of the NiCord unit, cryopreserved after selection, that contained a portion of the original graft's immunocompetent T cells and NK cells. Our hypothesis, which will need to be experimentally proven, is that this fraction provides support to NiCord-cultured HSPCs. This support, in combination with an enhanced HSPC dose following ex vivo expansion, allows NiCord to compete successfully for engraftment. Furthermore, the CD133- fraction provides the substrate for early cellular immune recovery via peripheral $\mathrm{T}$ cell expansion in the lymphodepleted host $(19,20)$.

Patients engrafted with NiCord were transplanted with a smaller $\mathrm{T}$ cell fraction compared with those receiving conventional single or dual UCB transplantation. As a result, there was a theoretic potential for compromised immunologic recovery in recipients with predominantly NiCord-derived donor chimerism. While the sample size was too small to draw firm conclusions, we were unable to detect quantitative differences in T cell, NK cell, B cell, or $\mathrm{T}$ cell receptor excision circle-positive cell (TREC) recovery between patients engrafted solely with the NiCord unit and those who received conventional dual UCB transplantation. Of note, 2 patients (patients 7 and 9) who were engrafted with the NiCord unit had prolonged dual cord blood $\mathrm{T}$ cell chimerism. Such a phenomenon was not observed in the control cohort. Interestingly, these 2 patients received cord blood units that were matched at 5 of 6 HLA loci. This was the highest degree of inter-cord blood matching among all study patients. The significance of this observation will require a larger study for confirmation.

The existence of CD34-, highly primitive HSPCs with longterm repopulating potential demonstrated in immunodeficient mice has been described $(21,22)$. The presence of these cells in a CD133- fraction has been neither confirmed nor refuted, raising the possibility that this nonexpanded fraction contributes to long-term engraftment of NiCord. We have transplanted high numbers of CD133- cells into NOD/ SCID mice as well as CD133-/CD3- cells into NSG mice and found no evidence of myeloid engraftment during a 6-month follow-up period (data not shown). Therefore, given the low cell dose of the transplanted CD133- fraction and the uncertain existence of long-term repopulating cells following CD133 depletion, the participation of this fraction in long-term engraftment we observed in NiCord recipients is unlikely, but cannot be ruled out.

This study is similar in design to 2 recently published studies of ex vivo-expanded cord blood HSPCs transplanted along with a second unmanipulated UCB unit. In the first of these trials, Delaney and colleagues transplanted cord blood that was enriched for $\mathrm{CD} 34^{+}$cells and expanded for 16 days in culture flasks or bags that were coated with Notch ligand (11). In the second study by de Lima and colleagues, 
A

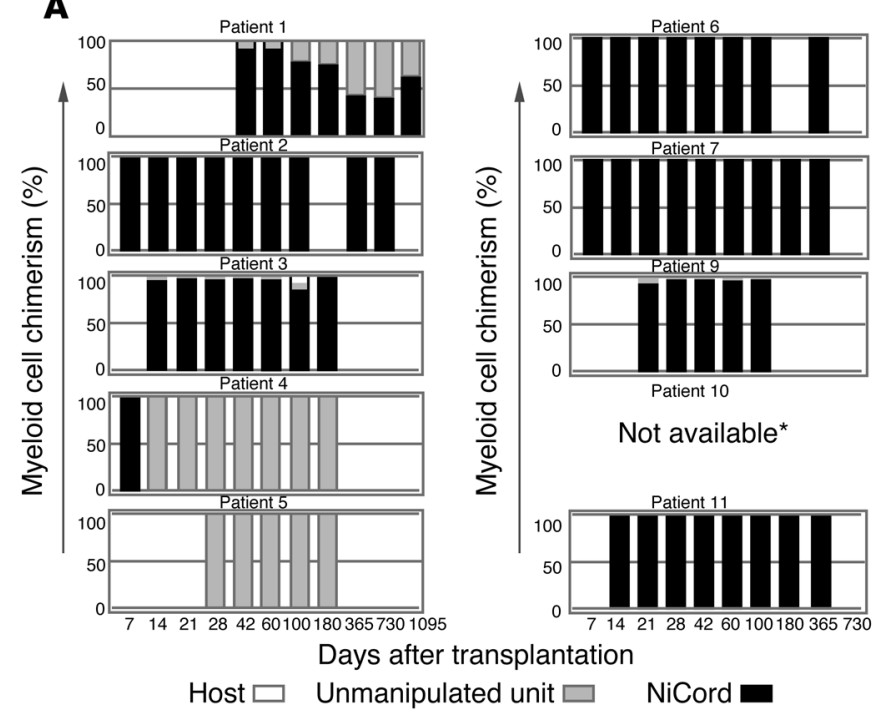

B

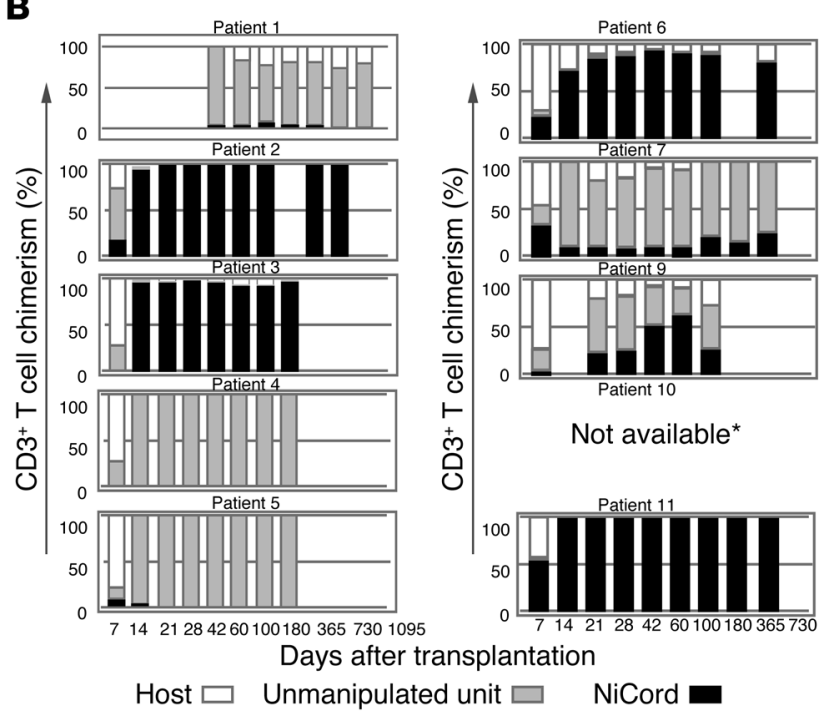

Figure 2

Myeloid $\left(\mathrm{CD} 15^{+}\right)$and T cell $\left(\mathrm{CD}^{+}\right)$chimerism measurements following transplantation. Bars demonstrate the median percentage of cells derived from the NiCord unit, the unmanipulated unit, and the host at serial time points following transplantation. Patient 8 experienced primary graft failure and was excluded. Whole-blood chimerism analysis was performed on samples from patient 10, showing $100 \%$ NiCord engraftment. Patients were censored at the time of documented relapse or death. *Whole-blood chimerism analysis demonstrated $100 \% \mathrm{NiCord}$ engraftment.

cord blood cells were cocultured for 14 days in flasks containing adherent mesenchymal precursor cells (12). In contrast to the present study, the expanded unit from these earlier studies was not supplemented with an infusion of immunocompetent $\mathrm{T}$ cells. The data from these studies clearly show that transplantation of ex vivo-cultured UCB stem cells shortens the time to hematopoietic recovery following myeloablative chemotherapy. This is accomplished by increasing the frequency of lineagecommitted short-term repopulating hematopoietic stem cells $(11,12)$. However, 6 months after transplantation, hematopoiesis derived from the expanded unit was negligible. In all cases, the coinfused unmanipulated cord blood unit provided long-term hematopoiesis. There are two potential explanations for the lack of long-term engraftment by the expanded unit in these prior studies. The first explanation is that HSPCs that possess longterm repopulating ability are lost during the ex vivo culture period. The second is that without coinfusion of immunocompetent $T$ cells, the ability of the expanded graft to compete successfully for long-term engraftment is lost.

The results of this trial provide justification for future studies to assess the safety and feasibility of transplantation of NiCord as a single, expanded UCB unit. Not only does NiCord expansion shorten the time to hematologic recovery, but it may also allow one to choose a smaller but better HLA-matched UCB unit for transplantation. This has the potential to mitigate the frequency and severity of GVHD and improve survival $(6,8,9)$.

In summary, this study demonstrates that transplantation of UCB progenitor cells that are cultured for 3 weeks using NiCord technology, along with a noncultured $\mathrm{T}$ cell fraction, provide rapid short-term engraftment and stable long-term multilineage hematopoiesis. Extended follow-up is needed to confirm the safety and durability of this cell product. NiCord-expanded UCB has the potential to broaden the accessibility and reduce the toxicity of UCB transplantation.

\section{Methods}

Patient and donor eligibility. Patients under the age of 65 years with hematologic malignancies and no available matched sibling or matched unrelated adult donor were eligible at Duke University School of Medicine or Loyola University Medical Center between December 2010 and August 2012. Eligibility required the availability of 1 cord blood unit containing at least $2.5 \times 10^{7}$ total nucleated cells per kilogram of the recipient's body weight. This unit was designated as the unmanipulated unit. The second unit, designated for NiCord expansion, contained at least $1.5 \times 10^{7}$ total nucleated cells per kilogram of the recipient's body weight. When 2 units with at least $2.5 \times 10^{7}$ total nucleated cells per kilogram of the recipient's body weight were available, the best-matched unit was assigned as the unmanipulated unit. The cord blood units were required to match the recipient at 4 or more HLA loci by intermediate-resolution typing for HLA class I alleles (A and B) and high-resolution typing for HLA class II DRB1 alleles. A minimum level of matching between the 2 cord blood units was not required. Cord blood units without cross-reactive donor-specific anti-HLA antibodies were prioritized for selection, but were not required for protocol participation.

NiCord production. The NiCord-designated (Gamida Cell) cryopreserved unit was delivered from the cord blood bank to a cGMP-compliant cell-processing facility (Lonza). The unit was thawed on day -21 of the stem cell transplantation and then underwent immunomagnetic bead selection for $\mathrm{CD}_{133^{+}}$cells (Clinimacs; Miltenyi Biotec). Following $\mathrm{CD} 133$ selection, the $\mathrm{CD} 133^{+}$and $\mathrm{CD} 34^{+}$cell content of the negative fraction was below $0.01 \%$ (data not shown). The CD133-, noncultured fraction was cryopreserved and shipped to the transplant center in a dry shipper maintained in a frozen state. Prior to cryopreservation, the cells were enumerated, immunophenotyped, and tested for viability and safety (limulus amebocyte lysate [LAL] endotoxin and sterility). The $\mathrm{CD}_{133^{+}}$fraction was tested for viability and purity and then suspended in MEM $\alpha$ supplemented with $10 \%$ FBS, 50 ng per milliliter each of Flt3 ligand, stem cell factor, thrombopoietin, IL-6 (R\&D Systems), and 2.5 millimoles nicotinamide (Vertellus) at a concentration of $1 \times 10^{4}$ cells per milliliter and seeded in culture bags (American Fluoroseal Corp.). 

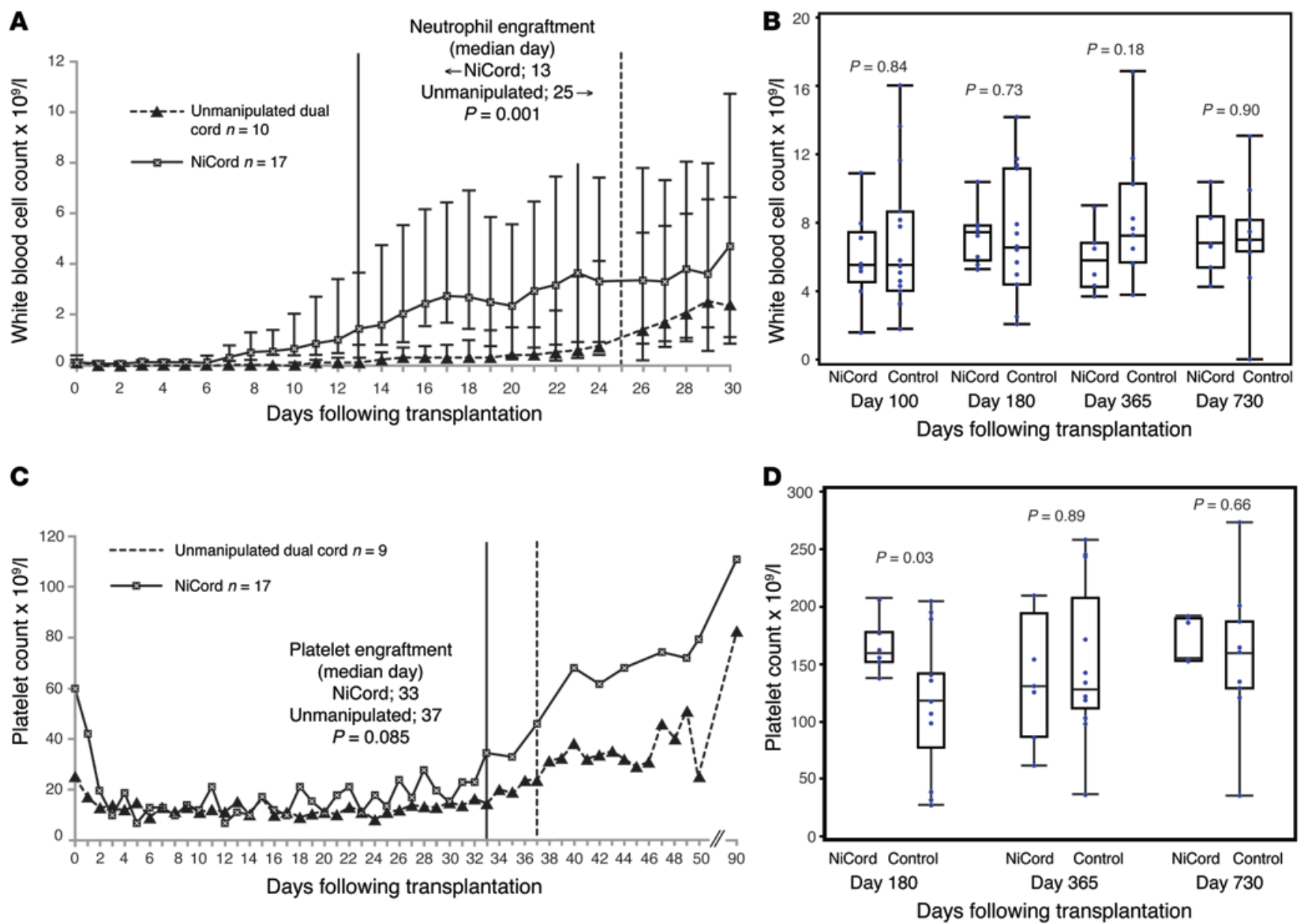

Figure 3

Hematopoietic recovery following transplantation. (A) Early white blood cell recovery with associated neutrophil engraftment and (B) durability of white blood cell count at late time points in patients transplanted with NiCord and in a historical control cohort. Bars represent the interquartile range. (C) Early platelet recovery and (D) durability of platelet count at late time points in patients transplanted with NiCord and in a historical control cohort. The historical control patients were transplanted with 2 unmanipulated UCB units.

The cultures were supplemented weekly with fresh medium. After $21 \pm 2$ days of culture, the cells were harvested, washed, and prepared for infusion in a transfusion PBS buffer. The fresh product was handdelivered to the transplant center at room temperature. Before release, the final product was tested for total number of cells, colony-forming unit content, and immunophenotype. Safety testing (gram staining, LAL endotoxin, and sterility) was repeated. All products were infused within the stability specification of 18 hours. The median time from harvest to infusion was 11.3 hours (range, $10-14$ hours).

Transplantation approach. The bone marrow-conditioning regimen consisted of 1,350-cGy TBI delivered in 9 fractions on days -9 to -5 , and fludarabine $40 \mathrm{mg} / \mathrm{m}^{2}$ was given on days -5 to -2 of the transplantation. An optional infusion of $60 \mathrm{mg} / \mathrm{kg}$ cyclophosphamide on days -4 and -3 was given at the discretion of the managing physician. Infusion of the NiCord and unmanipulated grafts was separated by a minimum of 2 hours and the infusion order alternated after every third patient. The noncultured CD133- fraction of the NiCord graft was thawed on the day of transplantation and infused after the cultured fraction. GVHD prophylaxis was provided by tacrolimus and mycophenolate mofetil starting 4 days before transplantation. Mycophenolate mofetil and tacrolimus were continued for a minimum of 60 days and 6 months following trans- plantation, respectively. G-CSF ( $5 \mu \mathrm{g} / \mathrm{kg}$ of the recipient's body weight) was given daily starting on day 1 following transplantation until the absolute neutrophil count exceeded 1,000 cells per microliter of blood. Patients were eligible for discharge from the hospital when the absolute neutrophil count exceeded 500 cells per microliter of blood.

Historical controls. Neutrophil, platelet, and immunologic recovery of study patients were compared with those of a cohort of 17 patients who enrolled in a preceding phase II study at the Duke Adult Blood and Marrow Transplant Program between April 2006 and January 2010 (14). To facilitate this analysis, we excluded patients from this control cohort who experienced primary graft failure or toxic death before engraftment. Patient characteristics are shown in Table 1. Patients received 2 unmanipulated UCB units each containing a minimum of $1.5 \times 10^{7}$ million nucleated cells per kilogram of the recipient's body weight. The cord blood units were required to match the recipient at 4 or more HLA loci by intermediate-resolution typing for HLA class I alleles (A and B) and high-resolution typing for HLA class II DRB1 alleles. A minimum level of matching between the 2 cord blood units was not required. The presence of donor-specific anti-HLA antibodies was not factored into the cord blood unit selection algorithm. The conditioning regimen, GVHD prophylaxis, and supportive care measures (including use of G-CSF) were identical to those of the current study cohort. 
Table 3

Clinical outcome of transplantation

\begin{tabular}{|c|c|c|c|c|c|c|c|c|}
\hline \multirow{2}{*}{$\begin{array}{l}\text { Patient } \\
\text { no. }\end{array}$} & \multirow{2}{*}{$\begin{array}{l}\text { Conditioning } \\
\text { regimen }\end{array}$} & \multirow{2}{*}{$\begin{array}{c}\text { Infusion } \\
\text { order }\end{array}$} & \multirow{2}{*}{$\begin{array}{l}\text { Engrafted } \\
\text { unit }\end{array}$} & \multicolumn{2}{|c|}{ Engraftment day } & \multicolumn{2}{|c|}{ GVHD } & \multirow[t]{2}{*}{ Clinical outcome } \\
\hline & & & & ANC $>500$ & Platelet $>\mathbf{2 0 , 0 0 0}$ & $\begin{array}{c}\text { Acute } \\
\text { (grades II-IV) }\end{array}$ & Chronic & \\
\hline 1 & $\mathrm{TBI} / \mathrm{flu}$ & $\begin{array}{l}\text { 1. Unmanip } \\
\text { 2. NiCord }\end{array}$ & $\begin{array}{c}\text { NiCord + } \\
\text { unmanipulated }\end{array}$ & 14 & 33 & Grade II & & Alive/CR (36 mo) \\
\hline 2 & $\mathrm{TBI} / \mathrm{flu}$ & $\begin{array}{l}\text { 1. Unmanip } \\
\text { 2. NiCord }\end{array}$ & NiCord & 11 & 30 & & & Alive/CR (31 mo) \\
\hline 3 & $\mathrm{TBI} / \mathrm{flu}$ & $\begin{array}{l}\text { 1. Unmanip } \\
\text { 2. NiCord }\end{array}$ & NiCord & 10 & 30 & & & $\begin{array}{l}\text { Death/relapse } \\
\quad(18 \mathrm{mo})\end{array}$ \\
\hline 4 & TBI/flu & $\begin{array}{l}\text { 1. NiCord } \\
\text { 2. Unmanip }\end{array}$ & Unmanipulated & 18 & 36 & & & Alive/CR (27 mo) \\
\hline 5 & TBI/flu & $\begin{array}{l}\text { 1. NiCord } \\
\text { 2.Unmanip }\end{array}$ & Unmanipulated & 26 & 49 & Grade II & $\begin{array}{l}\text { Moderate } \\
\text { (intestine) }\end{array}$ & Alive/CR (25 mo) \\
\hline 6 & TBI/flu & $\begin{array}{l}\text { 1. NiCord } \\
\text { 2. Unmanip }\end{array}$ & NiCord & 10 & 30 & & & Alive/CR (21 mo) \\
\hline 7 & TBI/flu & $\begin{array}{l}\text { 1. Unmanip } \\
\text { 2. NiCord }\end{array}$ & $\begin{array}{c}\text { NiCord }+ \\
\text { unmanipulated }\end{array}$ & 7 & 26 & Grade II & & Alive/CR (19 mo) \\
\hline 8 & $\mathrm{TBI} / \mathrm{flu}$ & $\begin{array}{l}\text { 1. Unmanip } \\
\text { 2. NiCord }\end{array}$ & Graft failure & - & - & & & Alive/CR (18 mo) \\
\hline 9 & TBI/flu & $\begin{array}{l}\text { 1. Unmanip } \\
\text { 2. NiCord }\end{array}$ & NiCord & 14 & 41 & Grade II & & $\begin{array}{l}\text { Death/relapse } \\
\quad(6 \mathrm{mo})\end{array}$ \\
\hline 10 & TBI/Cy/flu & $\begin{array}{l}\text { 1. NiCord } \\
\text { 2. Unmanip }\end{array}$ & NiCord & 18 & - & & & $\begin{array}{l}\text { Death/pneumonia } \\
\quad \text { (47 days) }\end{array}$ \\
\hline 11 & TBI/Cy/flu & $\begin{array}{l}\text { 1. Unmanip } \\
\text { 2. NiCord }\end{array}$ & NiCord & 7 & 33 & Grade II & Mild (skin) & Alive/CR (14 mo) \\
\hline $12^{A}$ & TBI/flu & 1. Unmanip & Unmanipulated & 18 & 35 & & & Alive/CR (30 mo) \\
\hline
\end{tabular}

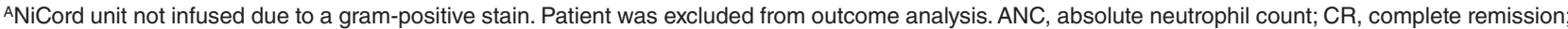
Cy, cyclophosphamide.

Laboratory and clinical assessments. Donor chimerism was performed on whole blood, $\mathrm{CD} 15^{+}$myeloid cells, and $\mathrm{CD}^{+} \mathrm{T}$ cells using quantitative analysis of informative microsatellite DNA sequences. Full donor chimerism was inferred when no detectable $\mathrm{CD} 15^{+}$myeloid or $\mathrm{CD} 3^{+}$lymphoid bands were observed from either the host or the second UCB unit. The

Product limit survival estimates

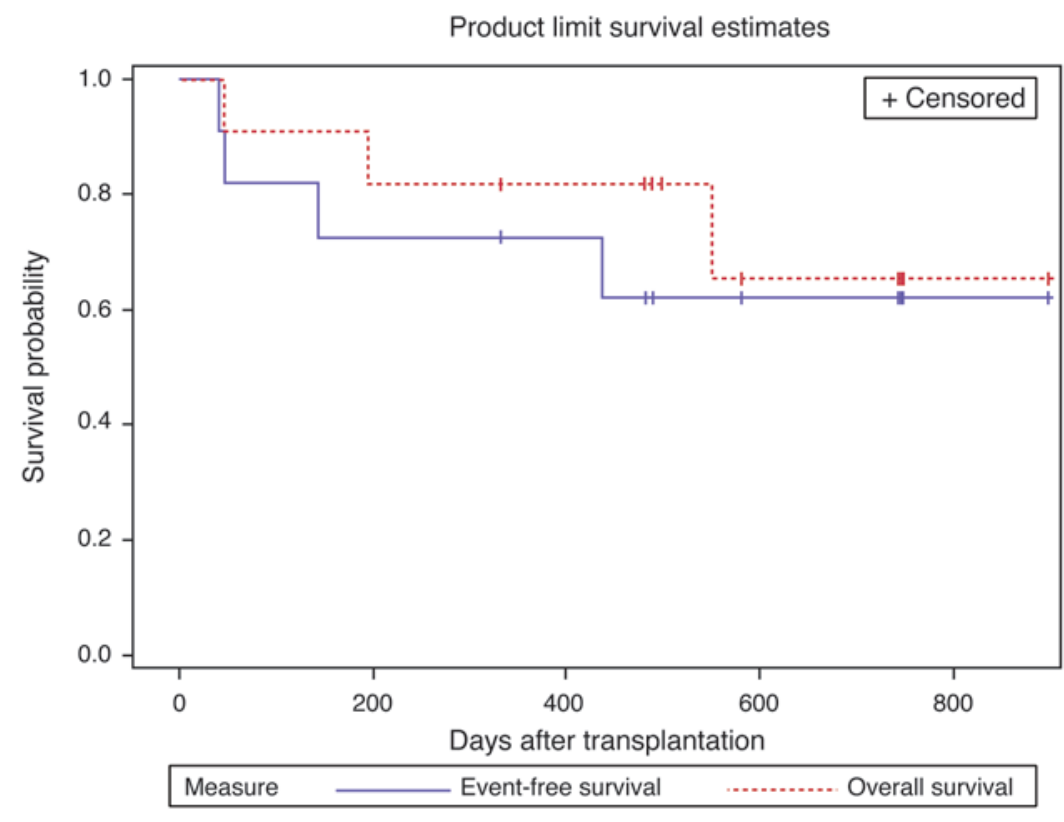

time to neutrophil engraftment was defined as the first of 3 consecutive days with an absolute neutrophil count of $0.5 \times 10^{9}$ per liter or higher and the time to platelet engraftment as the first of 7 consecutive days with a platelet count of $20 \times 10^{9}$ per liter or higher without platelet transfusion. Chronic GVHD was assessed using NIH consensus criteria.

\section{Figure 4}

Overall and event-free survival. Overall and eventfree survival for all subjects who received NiCordexpanded UCB stem cells. 
Statistics. Comparisons of the median time to neutrophil and platelet engraftment, cord blood graft characteristics, and hematologic recovery were performed using the Wilcoxon rank-sum test. For the initial hospitalization duration assessment, patients who died before hospital discharge were censored at the time of death, and patients who failed to engraft were excluded. A $P$ value less than or equal to 0.05 was considered significant. Overall and event-free survival rates were estimated using the Kaplan-Meier method. Events were defined as death, disease progression, or graft failure.

Study approval. The study was approved by the IRBs of both participating institutions and was conducted under an IND from the US Food and Drug Administration. All patients provided written informed consent. The study was conducted according to Declaration of Helsinki principles.

\section{Acknowledgments}

The authors thank the patients for their participation in the study. We acknowledge the important contribution of the nurses and staff of the adult stem cell transplantation programs at Duke University and Loyola University Medical Centers. We give special thanks to Ann Kaestner and Tiffany Bradshaw of the Duke Stem Cell Laboratory for their contribution in preparing the UCB units for transplantation. The trial was funded by Gamida Cell Ltd. This work was supported in part by grants from the National Cancer Institute, NIH (P01-CA047741-19, to M.E. Horwitz, N.J. Chao, and D.A. Rizzieri).

Received for publication January 7, 2014, and accepted in revised form April 17, 2014.

Address correspondence to: Mitchell E. Horwitz, Duke University School of Medicine, 2400 Pratt St., DUMC 3961, Durham, North Carolina 27710, USA. Phone: 919.668.1045; Fax: 919.668.1091; E-mail: mitchell.horwitz@duke.edu.
1. Brown JA, et al. Clearance of CMV viremia and survival after double umbilical cord blood transplantation in adults depends on reconstitution of thymopoiesis. Blood. 2010;115(20):4111-4119.

2. Kanda J, et al. Immune recovery in adult patients after myeloablative dual umbilical cord blood, matched sibling, and matched unrelated donor hematopoietic cell transplantation. Biol Blood Marrow Transplant. 2012;18(11):1664-1676.

3. Laughlin MJ, et al. Hematopoietic engraftment and survival in adult recipients of umbilical-cord blood from unrelated donors. N Engl J Med. 2001; 344(24):1815-1822.

4. Rocha V, et al. Transplants of umbilical-cord blood or bone marrow from unrelated donors in adults with acute leukemia. $N$ Engl J Med. 2004; 351(22):2276-2285.

5. Brunstein CG, et al. Allogeneic hematopoietic cell transplantation for hematologic malignancy: relative risks and benefits of double umbilical cord blood. Blood. 2010;116(22):4693-4699.

6. Eapen $M$, et al. Effect of donor-recipient HLA matching at HLA A, B, C, and DRB1 on outcomes after umbilical-cord blood transplantation for leukaemia and myelodysplastic syndrome: a retrospective analysis. Lancet Oncol. 2011;12(13):1214-1221.

7. Eapen M, et al. Outcomes after HLA-matched sibling transplantation or chemotherapy in children with B-precursor acute lymphoblastic leukemia in a second remission: a collaborative study of the Children's Oncology Group and the Center for International Blood and Marrow Transplant
Research. Blood. 2006;107(12):4961-4967.

8. Barker JN, Scaradavou A, Stevens CE. Combined effect of total nucleated cell dose and HLA match on transplantation outcome in 1061 cord blood recipients with hematologic malignancies. Blood. 2010;115(9):1843-1849.

9. Eapen $M$, et al. Impact of allele-level HLA matching on outcomes after myeloablative single unit umbilical cord blood transplantation for hematologic malignancy. Blood. 2014;123(1):133-140.

10. Scaradavou A, et al. Double unit grafts successfully extend the application of umbilical cord blood transplantation in adults with acute leukemia. Blood. 2013;121(5):752-758.

11. Delaney C, Heimfeld S, Brashem-Stein C, Voorhies H, Manger RL, Bernstein ID. Notch-mediated expansion of human cord blood progenitor cells capable of rapid myeloid reconstitution. Nat Med. 2010;16(2):232-236.

12. de Lima $M$, et al. Cord-blood engraftment with ex vivo mesenchymal-cell coculture. $N$ Engl J Med. 2012;367(24):2305-2315.

13. Peled $\mathrm{T}$, et al. Nicotinamide, a SIRT1 inhibitor, inhibits differentiation and facilitates expansion of hematopoietic progenitor cells with enhanced bone marrow homing and engraftment. Exp Hematol. 2012;40(4):342-355.

14. Kanda J, et al. Adult dual umbilical cord blood transplantation using myeloablative total body irradiation (1350 cGy) and fludarabine conditioning. Biol Blood Marrow Transplant. 2011;17(6):867-874.

15. Brunstein CG, et al. Umbilical cord blood transplan- tation after nonmyeloablative conditioning: impact on transplantation outcomes in 110 adults with hematologic disease. Blood. 2007;110(8):3064-3070.

16. Barker JN, et al. Transplantation of 2 partially HLA-matched umbilical cord blood units to enhance engraftment in adults with hematologic malignancy. Blood. 2005;105(3):1343-1347.

17. Gutman JA, et al. Single-unit dominance after double-unit umbilical cord blood transplantation coincides with a specific $\mathrm{CD}^{+} \mathrm{T}$-cell response against the nonengrafted unit. Blood. 2010;115(4):757-765.

18. Moretta A, et al. In vitro evaluation of graft-versus-graft alloreactivity as a tool to identify the predominant cord blood unit before double cord blood transplantation. Biol Blood Marrow Transplant. 2012;18(7):1108-1118.

19. Klein AK, et al. T-Cell recovery in adults and children following umbilical cord blood transplantation. Biol Blood Marrow Transplant. 2001; 7(8):454-466.

20. Mackall CL, et al. Age, thymopoiesis, and CD4+ T-lymphocyte regeneration after intensive chemotherapy. NEngl J Med. 1995;332(3):143-149.

21. Anjos-Afonso F, Currie E, Palmer HG, Foster KE, Taussig DC, Bonnet D. CD34(-) cells at the apex of the human hematopoietic stem cell hierarchy have distinctive cellular and molecular signatures. Cell Stem Cell. 2013;13(2):161-174.

22. Bhatia M, Bonnet D, Murdoch B, Gan OI, Dick JE. A newly discovered class of human hematopoietic cells with SCID-repopulating activity. Nat Med. 1998;4(9):1038-1045. 